\title{
GREEN'S FUNCTION OF THE CLAMPED PUNCTURED DISK
}

\author{
MITSURU NAKAI and LEO SARIO
}

(Received 1 December 1976)

\begin{abstract}
If a thin elastic circular plate $B:|z|<1$ is clamped (simply supported, respectively) along its edge $|z|=1$, its deflection at $z \in B$ under a point load at $\zeta \in B$, measured positively in the direction of the gravitational pull, is the biharmonic Green's function $\beta(z, \zeta)$ of the clamped plate $(\gamma(z, \zeta)$ of the simply supported plate, respectively). We ask: how do $\beta(z, \zeta)$ and $\gamma(z, \zeta)$ compare with the corresponding deflections $\beta_{0}(z, \zeta)$ and $\gamma_{0}(z, \zeta)$ of the punctured circular plate $B_{0}: 0<|z|<1$ that is "clamped" or "simply supported", respectively, also at the origin? We shall show that $\gamma(z, \zeta)$ is not affected by the puncturing, that is, $\gamma(\cdot, \zeta)=\gamma_{0}(\cdot, \zeta)$, whereas $\beta(\cdot, \zeta)$ is:

$$
\beta_{0}(z, \zeta)=\beta(z, \zeta)-16 \pi \beta(z, 0) \beta(\zeta, 0)
$$

on $B_{0} \times B_{0}$. Moreover, while $\beta(\cdot, \zeta)$ is of constant sign, $\beta_{0}(\cdot, \zeta)$ is not. This gives a simple counterexample to the conjecture of Hadamard [6] that the deflection of a clamped thin elastic plate be always of constant sign:

The biharmonic Green's function of a clamped concentric circular annulus is not of constant sign if the radius of the inner boundary circle is sufficiently small.

Earlier counterexamples to Hadamard's conjecture were given by Duffin [2], Garabedian [4], Loewner [7] and Szegö [9]. Interest in the problem was recently revived by the invited address of Duffin [3] before the Annual Meeting of the American Mathematical Society in 1974. The drawback of the counterexample we will present is that, whereas the classical examples are all simply connected, ours is not. In the simplicity of the proof, however, there is no comparison.
\end{abstract}

\section{Clamping and simple supporting}

First we make precise what we mean by clamping and simple supporting at the isolated point $O$. Denote by $B_{s}$ the annulus $s<|z|<1$ for $s \in(0,1)$. The corresponding biharmonic Green's function $\beta_{s}(z, \zeta)\left(\gamma_{8}(z, \zeta)\right.$, respectively) of the clamped (simply supported, respectively) annulus $B_{s}$ is characterized by

$$
\Delta^{2} \beta_{s}(\cdot, \zeta)=\delta_{\zeta} \quad\left(\Delta^{2} \gamma_{s}(\cdot, \zeta)=\delta_{\zeta} \text {, respectively }\right)
$$


on $B_{s}$, and

$$
\beta_{s}(\cdot, \zeta)=\frac{\partial}{\partial n} \beta_{s}(\cdot, \zeta)=0 \quad\left(\gamma_{s}(\cdot, \zeta)=\Delta \gamma_{s}(\cdot, \zeta)=0, \text { respectively }\right)
$$

on the boundary $\partial B_{s}$ of $B_{s}$. Here $\Delta$ is the Laplace-Beltrami operator $-\left(\partial^{2} / \partial x^{2}+\partial^{2} / \partial y^{2}\right), \delta_{\zeta}$ is the Dirac delta at $\zeta \in B_{s}$, and $\partial / \partial n$ denotes the inner normal derivative (for example, Bergman and Schiffer [1]). We will define $\beta_{0}(\cdot, \zeta)$ and $\gamma_{0}(\cdot, \zeta)$ as the limits of $\beta_{8}(\cdot, \zeta)$ and $\gamma_{s}(\cdot, \zeta)$, respectively, as $s \rightarrow 0$.

\section{Simply supported punctured disk}

Denote by $g_{s}(\cdot, \zeta)$ the harmonic Green's function of $B_{s}$ with pole $\zeta \in B_{s}$, and by $g(\cdot, \zeta)$ that of $B$. By the maximum principle and the Riemann removability theorem, $\left\{g(\cdot, \zeta)-g_{s}(\cdot, \zeta)\right\}$ converges decreasingly and uniformly to zero on each compact subset of $\bar{B}-0$ as $s \rightarrow 0$, and

$$
g(z, \zeta)=\frac{1}{2 \pi} \log \left|\frac{1-\bar{\zeta} z}{z-\zeta}\right|
$$

In view of (1) and (2), we have

$$
\gamma_{s}(z, \zeta)=\int_{B_{s}} g_{s}(w, z) g_{s}(w, \zeta) d u d v \quad(w=u+i v)
$$

on $B_{s} \times B_{s}$. On letting $s \rightarrow 0$ we see that

$$
\gamma_{0}(z, \zeta)=\lim _{s \rightarrow 0} \gamma_{s}(z, \zeta)
$$

exists uniformly on each compact subset of $B_{0} \times B_{0}$, and

$$
\gamma_{0}(z, \zeta)=\int_{B_{0}} g(w, z) g(w, \zeta) d u d v .
$$

On the other hand, since $\Delta^{2} \gamma(\cdot, \zeta)=\delta_{\zeta}$ on $B$ and $\gamma(\cdot, \zeta)=\Delta \gamma(\cdot, \zeta)=0$ on $\partial B$,

$$
\gamma(z, \zeta)=\int_{B} g(w, z) g(w, \zeta) d u d v .
$$

On comparing the right-hand sides of (5) and (6) we conclude that

$$
\gamma_{0}(z, \zeta)=\gamma(z, \zeta)
$$

on $B_{0} \times B_{0}$, that is, simple supporting at a single point does not have any effect on the deflection of a simply supported disk. This result agrees with physical intuition: if we place the tip of a needle under a very thin plate that is simply supported along its periphery, and then put a point load on the plate, the plate will be pierced by the needle. 


\section{Clamped punctured disk}

In contrast with the above, what happens to $\beta_{0}(\cdot, \zeta)$ is somewhat surprising. Denote by $H_{s}(\cdot, \zeta)=\Delta \beta_{s}(\cdot, \zeta)$ the $\beta$-density of $\beta_{s}(\cdot, \zeta)$. It is readily deduced from Stokes' formula that $H_{s}(\cdot, \zeta) \perp H_{2}\left(B_{8}\right)$, that is

$$
\int h(w) H_{s}(w, \zeta) d u d v=0
$$

for any $h$ in the class $H_{2}\left(B_{s}\right)$ of square integrable harmonic functions on $B_{s}$ (cf. [8]). As consequences of (8) we easily obtain

$$
\begin{aligned}
& \beta_{s}(z, \zeta)=\int_{B_{s}} H_{s}(w, z) H_{s}(w, \zeta) d u d v \\
& \left\|H_{l}(\cdot, \zeta)-H_{s}(\cdot, \zeta)\right\|^{2}=\left\|H_{l}(\cdot, \zeta)\right\|^{2}-\left\|H_{s}(\cdot, \zeta)\right\|^{2} \quad(0<t<s<1) \\
& \left|\beta_{l}(z, \zeta)-\beta_{s}(z, \zeta)\right| \leqslant\left\|H_{l}(\cdot, z)-H_{s}(\cdot, z)\right\| \cdot\left\|H_{l}(\cdot, \zeta)-H_{s}(\cdot, \zeta)\right\| \\
& \left\|H_{s}(\cdot, \zeta)\right\| \leqslant\|g(\cdot, \zeta)\|
\end{aligned}
$$

where $\|\cdot\|$ is the $L_{2}$-norm on $B$ and functions here and hereafter are defined to be zero outside their genuine domains of definition. It follows that

$$
\beta_{0}(z, \zeta)=\lim _{s \rightarrow 0} \beta_{s}(z, \zeta)
$$

exists uniformly on each compact subset of $\bar{B}-O$. If we denote by

$$
H_{0}(\cdot, \zeta)=\Delta \beta_{0}(\cdot, \zeta)
$$

the $\beta$-density of $\beta_{0}(\cdot, \zeta)$, then by (8) and (9),

$$
\begin{aligned}
& H_{0}(\cdot, \zeta) \perp H_{2}\left(B_{0}\right), \\
& \lim _{s \rightarrow 0}\left\|H_{0}(\cdot, \zeta)-H_{s}(\cdot, \zeta)\right\|=0, \\
& \beta_{0}(z, \zeta)=\int_{B_{0}} H_{0}(w, z) H_{0}(w, \zeta) d u d v=\int_{B_{0}} H_{0}(w, z) K(w, \zeta) d u d v,
\end{aligned}
$$

where $K(\cdot, \zeta)$ is any square integrable function on $B_{0}$ with $\Delta K(\cdot, \zeta)=\delta_{\zeta}$ on $B$.

\section{Clamped disk}

The function $\beta(\cdot, \zeta)$ is defined by $\Delta^{2} \beta(\cdot, \zeta)=\delta_{\zeta}$ on $B$ and $\beta(\cdot, \zeta)=\partial \beta(\cdot, \zeta) / \partial n=0$ on $\partial B$. An explicit expression for $\beta(\cdot, \zeta)$ is known (for example, Garabedian [5]):

$$
\beta(z, \zeta)=\frac{1}{8 \pi}\left[|z-\zeta|^{2} \log \left|\frac{z-\zeta}{1-\bar{\zeta} z}\right|+\frac{1}{2}\left(|z|^{2}-1\right)\left(|\zeta|^{2}-1\right)\right]
$$


on $B \times B$. Our immediate aim is to express $\beta_{0}(z, \zeta)$ in terms of $\beta(z, \zeta)$. The basis of our computation is the first relation (11) and its counterpart $H(\cdot, \zeta) \perp H_{0}(B)$, where $H(\cdot, \zeta)=\Delta \beta(\cdot, \zeta)$ is the $\beta$-density of $\beta(\cdot, \zeta)$. The latter orthogonality relation implies that

$$
\beta(z, \zeta)=\int_{B} H(w, z) H(w, \zeta) d u d v=\int_{B} H(w, z) g(w, \zeta) d u d v
$$

on $\boldsymbol{B} \times \boldsymbol{b}$. Since $H^{\prime}(\cdot, \zeta)-H_{0}(\cdot, \zeta)$ is harmonic on $B_{0}$ and square integrable over $B_{0}$, we have

$$
H\left(r e^{i \theta}, \zeta\right)-H_{0}\left(r e^{i \theta}, \zeta\right)=a g(r)+b+\sum_{n=1}^{\infty}\left(\sum_{m=1}^{2} c_{n m} S_{n m}(\theta)\right) r^{n}
$$

with uniform convergence on each compact subset of $\bar{B}-0$. Here $a, b$ and $c_{n m}$ are constants, $g(r)=g(r, 0)=-(1 / 2 \pi) \log r, S_{n 1}(\theta)=\cos n \theta$, and $S_{n 2}(\theta)=\sin n \theta$ for $n=1,2, \ldots$. We denote by $(\cdot, \cdot)$ the inner product on $L_{2}(B)$ and by $\|\cdot\|_{1}$ the norm on $L_{1}(B)$. Since $h_{n m}\left(r e^{i \theta}\right)=S_{n m}(\theta) r^{n}$ is in the class $H_{2}(B) \subset H_{2}\left(B_{0}\right)$, and $\left\|h_{n m}\right\| \neq 0$,

$$
c_{n m}\left\|h_{n m}\right\|^{2}=\left(H(\cdot, \zeta)-H_{0}(\cdot, \zeta), h_{n m}\right)=0
$$

and $c_{n m}=0$ for every $n$ and $m$. Observe that

$$
\begin{aligned}
& \left(H(\cdot, \zeta)-H_{0}(\cdot, \zeta), 1\right)=a\|g\|_{1}+b \pi, \\
& \left(H(\cdot, \zeta)-H_{0}(\cdot, \zeta), g\right)=a\|g\|^{2}+b\|g\|_{1} .
\end{aligned}
$$

By virtue of $1 \in H_{2}(B) \subset H_{2}\left(B_{0}\right)$ and $g \in H_{2}\left(B_{0}\right)$ (but $g \notin H_{2}(B)$ ), these equations take the form

$$
\begin{aligned}
\|g\|_{1} a+\pi b & =0, \\
\|g\|^{2} a+\|g\|_{1} b & =\beta(\zeta, 0) .
\end{aligned}
$$

In view of $\|g\|_{1}=1 / 4$ and $\|g\|^{2}=1 / 8 \pi$, we obtain $a=16 \pi \beta(\zeta, 0)$ and $b=-4 \beta(\zeta, 0)$. By (14), we conclude that

$$
H_{0}(\cdot, \zeta)=H(\cdot, \zeta)-16 \pi \beta(\zeta, 0) g(\cdot, 0)+4 \beta(\zeta, 0)
$$

on $B_{0}$. We take the inner product of each side of $(15)$ with $H(\cdot, z)$ and obtain by $(13)$ the following main identity of the present study:

$$
\beta_{0}(z, \zeta)=\beta(z, \zeta)-16 \pi \beta(z, 0) \beta(\zeta, 0)
$$

on $B_{0} \times B_{0}$. This is the required representation of $\beta_{0}$ in terms of $\beta$.

By (12), $\beta(z, \zeta)>0$ on $B \times B$, and a fortiori,

$$
\beta_{0}(z, \zeta)<\beta(z, \zeta)
$$


on $B_{0} \times B_{0}$. Thus adding to the clamping at the periphery, the clamping at a single point $O$ does have a noticeable effect on the resulting deflection. Compared with the case of $\gamma_{0}$, this result is quite intriguing.

We now analyse the boundary behavior of $\beta_{0}$ in some more detail, with a view on our main identity (16).

\section{Boundary behaviour}

By (12) and (16), we have $\Delta^{2} \beta_{0}(\cdot, \zeta)=\delta_{\zeta}$ on $B_{0}$ and $\beta_{0}(\cdot, \zeta)=\partial \beta_{0}(\cdot, \zeta) / \partial n=0$ on $\partial B:|z|=1$. Thus both clamping conditions are satisfied at the outer boundary $\partial B$. By $(12), \beta(0,0)=1 / 16 \pi$, and by $(16)$ and the symmetry of $\beta$,

$$
\begin{aligned}
\beta_{0}(0, \zeta) & =\lim _{z \rightarrow 0} \beta_{0}(z, \zeta) \\
& =\beta(0, \zeta)-16 \pi \beta(0,0) \beta(\zeta, 0)=0,
\end{aligned}
$$

that is, the first clamping condition is satisfied at the inner boundary $z=0$.

We proceed to examine the second condition. Denote by $\partial / \partial n_{\theta}$ the directional derivative in the direction $e^{i \theta}$, that is,

$$
\frac{\partial}{\partial n_{\theta}} \beta_{0}(0, \zeta)=\lim _{t \rightarrow+0} \frac{\beta_{0}\left(t e^{i \theta}, \zeta\right)-\beta_{0}(0, \zeta)}{t} .
$$

Again by (16),

$$
\frac{\partial}{\partial n_{\theta}} \beta_{0}(0, \zeta)=\frac{\partial}{\partial n_{\theta}} \beta(0, \zeta)-16 \pi \beta(\zeta, 0) \frac{\partial}{\partial n_{\theta}} \beta(0,0) .
$$

Since $\beta\left(t e^{i \theta}, 0\right)=(8 \pi)^{-1}\left[t^{2} \log t+\frac{1}{2}\left(t^{2}-1\right)\right]$ for $t>0$, we have

$$
\frac{\partial}{\partial n_{\theta}} \beta(0,0)=\lim _{t \rightarrow+0} \frac{\beta\left(t e^{i \theta}, 0\right)-\beta(0,0)}{t}=0
$$

and, therefore,

$$
\frac{\partial}{\partial n_{\theta}} \beta_{0}(0, \zeta)=\frac{\partial}{\partial n_{\theta}} \beta(0, \zeta) .
$$

By (12) we see that $\beta(z, \zeta)$ is real analytic in the neighbourhood $|z|<|\zeta|$ of $z=0$ and $a$ fortiori,

$$
\frac{\partial}{\partial n_{\theta}} \beta(0, \zeta)=\left[\frac{\partial}{\partial t} \beta\left(t e^{i \theta}, \zeta\right)\right]_{l=0} .
$$

Using the explicit representation (12) for $\beta(z, \zeta)$, we obtain by direct calculation

$$
\frac{\partial}{\partial n_{\theta}} \beta_{0}(0, \zeta)=\frac{1}{8 \pi}|\zeta|\left(|\zeta|^{2}-2 \log |\zeta|-1\right) \cos (\theta-\arg \zeta) \text {. }
$$

Thus the "normal derivative" of $\beta_{0}(z, \zeta)$ at $z=0$ does not vanish identically, and the second clamping condition is not satisfied. However, this failed "clamping" will conveniently serve to disprove Hadamard's conjecture, as we shall now see. 


\section{Hadamard's conjecture}

Hadamard [6] conjectured that the Green's function of a clamped thin elastic plate cannot take on negative values. We give here a simple counterexample based on $\beta_{0}(z, \zeta)$. Observe that for any $\zeta \in B_{0}$,

$$
|\zeta|^{2}-2 \log |\zeta|-1>0
$$

From this and (20) we see that

$$
\left.\operatorname{sign} \frac{\partial \beta_{0}(0, \zeta)}{\partial n_{\theta}}\right|_{\theta=\pi+\arg \zeta} \neq\left.\operatorname{sign} \frac{\partial \beta_{0}(0, \zeta)}{\partial n_{\theta}}\right|_{\theta=\arg \zeta}=+1 .
$$

This means that $\beta_{0}(z, \zeta)$ takes values of opposite sign on line segments

$$
\{z ; 0<|z|<\sigma, \arg z=\pi+\arg \zeta\} \text { and }\{z ; 0<|z|<\sigma, \arg z=\arg \zeta\}
$$

for a sufficiently small $\sigma \in(0,1)$. This rather agrees with our intuition provided $\beta_{0}(0, \zeta)=0$, a fact which, however, is not clear a priori.

By (10) we see that $\beta_{s}(\cdot, \zeta)$ converges to $\beta_{0}(\cdot, \zeta)$ uniformly on each compact subset of $B_{0}$ and therefore, $\beta_{s}(\cdot, \zeta)$ takes on values of nonconstant sign along with $\beta_{0}(\cdot, \zeta)$ if $s>0$ is sufficiently small. Thus we have the following

Counterexample to Hadamard's Conjecture. The Green's function of a clamped concentric circular annulus is of nonconstant sign if the radius of the inner boundary circle is sufficiently small.

\section{Acknowledgement}

The work was sponsored by the U.S. Army Research Office, Grant DA-ARO31-124-73-G39, University of California, Los Angeles. MOS Classification 31 B 30.

\section{REFERENCES}

[1] S. Bergman and M. Schiffer, Kernel functions and elliptic differential equations in mathematical physics. (Academic Press, New York, 1953, 432 pp.).

[2] R. J. Duffin, "On a question of Hadamard concerning super-biharmonic functions", J. Math. Physics 27 (1949), 253-258.

[3] R. J. Duffin, "Some problems of mathematics and science", Bull. Amer. Math. Soc. 80 (1974), 1053-1070.

[4] P. R. Garabedian, “A partial differential equation arising in conformal mapping”, Pacific J. Math. 1 (1951), 485-524.

[5] P. R. Garabedian, Partial differential equations. (Wiley, New York-London-Sydney, 1967, 672 pp.). 
[6] J. Hadamard, "Mémoire sur le problème d'analyse relatif à l'équilibre des plaques élastiques encastrées", Mémoires présentés par divers savants étrangers à l'Académie des Sciences, 33 (1908), 515-629.

[7] C. Loewner, "On generalization of solutions of the biharmonic equation in the plane by conformal mapping", Pacific J. Math. 3 (1953), 417-436.

[8] M. Nakai and L. Sario, "A strict inclusion related to biharmonic Green's functions of clamped and simply supported bodies", Ann. Acad. Sci. Fenn. A.I.3, 1977, 53-58.

[9] G. Szegö, "Remark on the preceding paper by Charles Loewner", Pacific J. Math. 3 (1953), 437-446.

Department of Mathematics

Nagoya Institute of Technology

Gokiso, Showa, Nagoya

Japan

and

Department of Mathematics

University of California

Los Angeles, California

U.S.A. 